Document downloaded from:

http://hdl.handle.net/10251/103252

This paper must be cited as:

Rincon, J.; De La Prieta-Pintado, F.; Zanardini, D.; Julian Inglada, VJ.; Carrascosa Casamayor, C. (2017). Influencing over people with a social emotional model. Neurocomputing. 231:47-54. doi:10.1016/j.neucom.2016.03.107

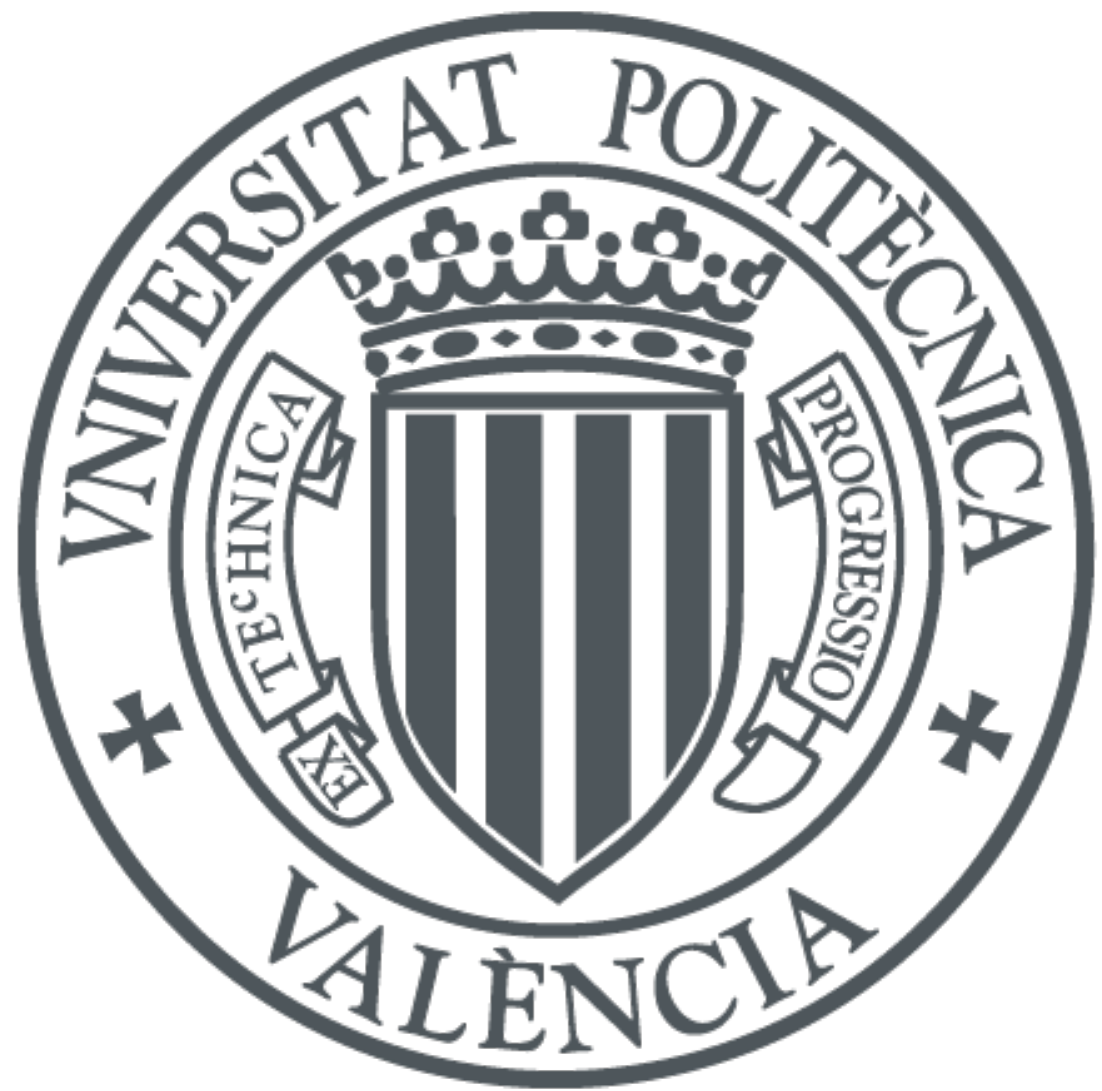

The final publication is available at

https://doi.org/10.1016/j.neucom.2016.03.107

Copyright Elsevier

Additional Information 


\title{
Influencing over People with a Social Emotional Model
}

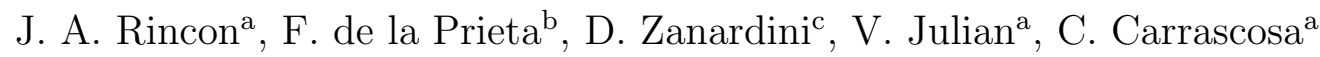 \\ ${ }^{a}$ Universitat Politècnica de València \\ Departamento de Sistemas Informáticos y Computación (DSIC) \\ Camino de Vera $s / n$, Valencia, Spain \\ \{jrincon,vinglada, carrasco\}@dsic.upv.es \\ ${ }^{b}$ Universitat Politècnica de València \\ Department of Computer Science - University of Salamanca \\ fer@usal.es \\ ${ }^{c}$ Departamento de Inteligencia Artificial, Universidad Politecnica de Madrid, Spain \\ damiano@fi.upm.es
}

\begin{abstract}
This paper presents an approach of a social emotional model, which allows to extract the social emotion of a group of intelligent entities. The emotional model $P A D$ allows to represent the emotion of an intelligent entity in 3-D space, allowing the representation of different emotional states. The social emotional model presented in this paper uses individual emotions of each one of the entities, which are represented in the emotional space $P A D$. Using a social emotional model within intelligent entities allows the creation of more real simulations, in which emotional states can influence decision-making. The result of this social emotional mode is represented by a series of examples, which are intended to represent a number of situations in which the emotions of each individual modify the emotion of the group. Moreover, the paper introduces an example which employs the proposed model in order to learn and predict future actions trying to influence in the social emotion of a group of people.
\end{abstract}

Keywords:

Multi-Agent systems, Neural Networks, Emotional Software Agents

\section{Introduction}

In the 80 s decade, Human-Computer Interaction (HCI) appeared as a new field giving access to the new digital technologies and converting all the 
people in potential users without any knowledge about computers. During last decades, HCI has involved information interchange between people and computers using some kind of dialogue, like programming languages and information interchange platforms. These platforms have included from input devices such as keyboards and optical mouses to output devices as the own computer screens.

Lastly, cognitive psychology integration within the HCI field leads to adopt new forms of information processing and to better understanding how people communicate with the devices. Nevertheless, in spite of the accessibility solutions presented by HCIs, user interfaces were very limited. As a result, the discipline has adopted other research subjects focused in usability, ergonomics trying to build new interfaces and allowing a more natural interaction between humans and machines.

These research subjects have made appear new interaction paradigms created by the mobile computing, portable and ubiquitous. They have incorporated devices to communicate directly with the physical world such as movement and gestures capture through the Kinect [1] and even user biosignals capture through the $M Y O$ and Emotiv devices [2], [3]. The idea is that machines will not only receive orders from users but also they will perceive their emotional states or behaviors using all this information to execute the different actions [4], [5].

The information increase generated by the new ways of interaction has made appear the need of using other computational toolkits to analyse and process information to benefit users. Artificial Intelligence tools such as pattern recognition ones, machine learning, and multi-agent systems (MAS) allow the development of this kind of complex tasks, creating adaptive environments to human needs to improve his welfare and life quality.

Human beings manage themselves in different environments, either in the working place, at home or in public places. At each one of these places we perceive a wide range of stimuli, that interfere in our commodity levels modifying our emotional levels. For instance, the high levels of noise or the temperature conditions may produce stress situations. Before each one of these stimuli, humans answer varying our face gestures, body or bio-electrical ones. These variations in our emotional states could be used as information useful for machines. Nevertheless, it is needed that the machines will have the capability of interpreting or recognizing such variations. This is the reason for implementing emotional models to represent the different emotions.

Emotional models such as $O C C$ [6] presented by Ortony, Clore \& Collins 
and the PAD model [7] are the most used ones to detect or simulate emotional states. Nevertheless, these models don't allow the execution of intelligent decisions based on the emotional state perception. Between these toolkits, we can find MAS, which are able to modify their behavior based on the emotional state perception. This way, it is obtained that the agent being part of the MAS contains an emotional model able of interpreting and/or emulating different emotional states. To detect emotional states, it is needed to include pattern recognition algorithms, automatic learning contributing to the decision making to execute an action. For instance, if an agent detects that the user presents an emotional state of sadness, it is able to counter that emotional state by executing actions trying to modify it. This way a clean and transparent human-machine interaction is obtained. However, this situation is only valid for a lonely entity inside the environment. The incorporation of more entities inside the environment (multiple emotions) is not contemplated by current emotional models.

The goal of this work is to give an approach to a social emotional model including multiple emotions between humans and agents. Our model uses as base the $P A D$ emotional model to represent the social emotion of a group. Moreover, the paper introduces a case study where the social emotion is used for predicting next actions in order to improve the emotional state of a group of people. Concretely, the case study has been developed simulating a bar, where there is a DJ in charge of playing music and a specific number of persons listening that music. The main goal of the DJ is to play music making that all the people within the bar are mostly as happy as possible. This proposed application engages Ambient Intelligence (AmI) and Ubiquitous Computing $(U C)$ involving humans and helping to improve their living conditions. This kind of applications involves the interaction between humans and agents, being these responsible for a continuous monitoring of the different emotional states. This is mainly due to the influence that music can have on people's moods. This influence has already been studied [8] analyzing how different musical genres can influence people's emotions. Existing works take into account the social and cultural importance of music which influences positively or negatively on people behavior [9],[10], [11].

The rest of the paper is structured as follows: Section 2 introduces related work; Section 3 presents the social emotional model; Section 4 describes the case study introducing some illustrative situations and analysing the obtained results; finally, Section 5 includes some conclusions. 


\section{Previous approaches}

This section presents an introduction to the emotional models $O C C$ and $P A D$. The goal is to give a general view of both emotional models.

\subsection{Ortony, Clore \& Collins: $O C C$}

The $O C C$ model designed by Ortony, Clore \& Collins is a model frequently used in applications where an emotional state can be detected or simulated. This has allowed to create applications to emulate emotions in virtual humans [12] and to create agents reacting to stress situations [13].

The $O C C$ model specifies 22 emotional categories, which are divided into five processes: 1) the classification of the events, the action or the found object, 2) the quantification of the affected emotions intensity, 3) the interaction between the just generated emotion with the existing ones, 4) the cartography of the emotional state of one emotional expression and 5) is the one expressed by the emotional state [14]. In $O C C$ model is observed. These processes define the whole system, where the emotional states represent the way of perceiving our environment (objects, persons, places) and, at the same time, influencing in our behaviour positively or negatively [15]. However, the $O C C$ model utilization presents one complication due mainly to his high dimensionality.

\subsection{PAD Model}

The $P A D[16]$ is a simplified model of the $O C C$ model, since the $O C C$ model represents the emotion using eleven dimensions whilst the $P A D$ only uses three. This reduction of dimensionality allows to do mathematical operations faster and to represent the emotion as a vector in the $\mathbb{R}^{3}$ space. In this representation, the values that compose the emotion are usually normalized in the range $[-1,1]$, and correspond to the three components conforming the emotional model (Pleasure, Arousal, Dominance). Each one of these components allow to influence over the emotional state of an individual in a positive or negative way. This influence evaluates the emotional predisposition of such individual, modifying in this way his emotional state. The Pleasure-Displeasure Scale measures how pleasant an emotion may be. For instance both anger and fear are unpleasant emotions, and score high on the displeasure scale. However joy is a pleasant emotion. This dimension is usually limited to 16 specific values. ( [17], pp. 39-53). The ArousalNonarousal Scale measures the intensity of the emotion. For instance while 
both anger and rage are unpleasant emotions, rage has a higher intensity or a higher arousal state. However boredom, which is also an unpleasant state, has a low arousal value. This scale is usually restricted to 9 specific values([17], pp. 39-53). The Dominance-Submissiveness Scale represents the controlling and dominant nature of the emotion. For instance while both fear and anger are unpleasant emotions, anger is a dominant emotion, while fear is a submissive emotion.

As have been presented above, the existing emotional models are thought to detect and/or simulate human emotions for a lonely entity. That is, it is not taken into account the possibility of having multiple emotions inside an heterogeneous group of entities, where each one of such entities have the capability of detecting and/or emulating one emotion. The need of detecting the emotion of an heterogeneous group of entities can be reflected in the different applications that could be obtained. With the appearance of the different smart devices, ubiquituous computation and ambient intelligent, emotional states turn into valuable information, allowing to develop applications that help to improve the human being life quality. Therefore, it is needed to create a new model that allows to detect the emotion of a group.

\section{Social Emotional Model Based on the PAD model}

This section proposes a model of social emotion based on the PAD emotional model. This model will represent the social emotion of a heterogeneous group of entities capable of expressing and/or communicate emotions. To define a model of social emotion, it is necessary first to define the representation of an emotional state of an agent on the PAD model. The emotion of an agent $a g_{i}$ is defined as a vector in a space $\mathbb{R}^{3}$, represented by three components that make up the $P A D$ emotional model: $P=$ Pleasure, $A=$ Arousal and $D=$ Dominance. Each one of these elements allow to represent the emotion of each agent $a g_{i}$. The variation of each component allows to modify the emotional state of the agent (Equation 1).

$$
\vec{E}\left(a g_{i}\right)=\left(P_{i}, A_{i}, D_{i}\right)
$$

A first approach to a social emotion representation of a group of $n$ agents $A g=\left\{a g_{1}, a g_{2}, \ldots, a g_{n}\right\}$ is obtained by averaging their $P, A, D$ values (Equation 2). This average will enable us to determine where the central emotion $(C E)$ of this group of agents and be visualized in the $P A D$ space. 


$$
\bar{P}=\frac{\sum_{i=1}^{n} P_{i}}{n}, \bar{A}=\frac{\sum_{i=1}^{n} A_{i}}{n}, \bar{D}=\frac{\sum_{i=1}^{n} D_{i}}{n}
$$

The final result is a vector in the space $\mathbb{R}^{3}$ which is the core emotion or $\overrightarrow{C E}(A g)$ of a group of agents (Equation 3).

$$
\overrightarrow{C E}(A g)=[\bar{P}, \bar{A}, \bar{D}]
$$

The $\overrightarrow{C E}(A g)$ by itself is not enough to represent the social emotion of a group of agents, since there may be different groups of agents with the same central emotion but in a very different emotional situation. Figures 1 and $2^{1}$, show two different situations where the central emotion is the same. In Figure 1, the emotional states of a group of agents is observed with $\overrightarrow{C E}(A g)=[0.0,0.22,0.45]$. As can be observed, the individual emotional states of these agents are subdivided into two very different groups. In fact, they may represent, for instance, a group of people which are seeing a football match that has just finished, having supporters from the two teams. On the other hand, Figure 2 shows the individual emotional states of another group of agents with completely different emotions. Although it can also be observed two different groups, their emotions are not as oppossed as in the previous figure. Nevertheless, as before commented, the two examples represented in Figure 1 and Figure 2 generate the same central emotion.

Clearly, the $\overrightarrow{C E}(A g)$ is not enough to represent the social emotion of an agent group. As it can be seen in the previous example the emotions of an agents group can be very different but have the same $\overrightarrow{C E}$. This is why it is necessary to introduce some measurement about the distance of the agents with respect to the $\overrightarrow{C E}$. To do this we include the definition of the maximum distances of agent emotions respect to $\overrightarrow{C E}(A g)$. In order to calculate the maximum distances, the Euclidean distance (Equation 4, 5, 6) is used as follows.

$$
\begin{aligned}
& m_{P}(A g)=\max \left(\sqrt{\left(P_{i}-\bar{P}(A g)\right)^{2}}\right), \forall a g_{i} \in A g / \vec{E}\left(a g_{i}\right)=\left[P_{i}, A_{i}, D_{i}\right] \\
& m_{A}(A g)=\max \left(\sqrt{\left(A_{i}-\bar{A}(A g)\right)^{2}}\right), \forall a g_{i} \in A g / \vec{E}\left(a g_{i}\right)=\left[P_{i}, A_{i}, D_{i}\right]
\end{aligned}
$$

\footnotetext{
${ }^{1}$ This is a graphical representation of the emotional states of a group of agents according to the values of the PAD model in a space $\mathbb{R}^{3}$. Each red triangle represents the emotional state of an individual agent and the green triangle represents the central emotion $\overrightarrow{C E}(A g)$ of the group of agents.
} 


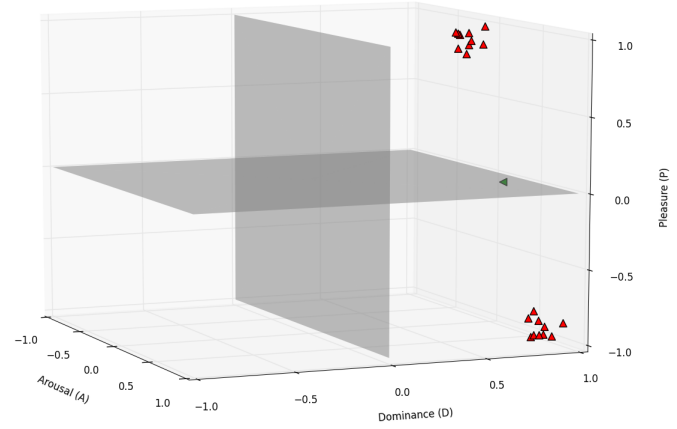

Figure 1: Representation of a group of agent's emotions with two subgroups completely opposite.

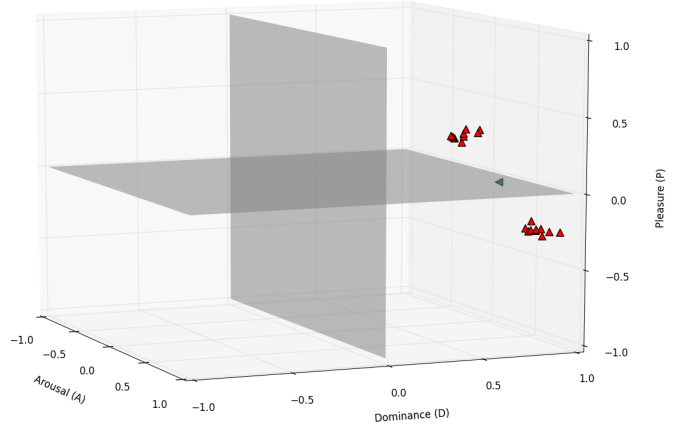

Figure 2: Representation of a group of agent's emotions with two subgroups with more nearest emotions.

$$
m_{D}(A g)=\max \left(\sqrt{\left(D_{i}-\bar{D}(A g)\right)^{2}}\right), \forall a g_{i} \in A g / \vec{E}\left(a g_{i}\right)=\left[P_{i}, A_{i}, D_{i}\right]
$$

The results of these equations can be represented as a vector of maximum distances (Equation 7).

$$
\vec{m}(A g)=\left[m_{P}(A g), m_{A}(A g), m_{D}(A g)\right]
$$

The $\vec{m}(A g)$ can indicate if there exist agents having their emotional state far away from the central emotion. From a graphical perspective it is also possible to use these maximum distances to plot an enveloping which encapsulates all emotions, allowing the limit of all the agents to be defined. 
To represent this enveloping shape of emotions an ellipsoid as a geometric figure was used. This ellipsoid (Figure 3$)^{2}$ can be adapted to represent different emotional states, which allows a dynamical way for displaying the social emotion of a group.

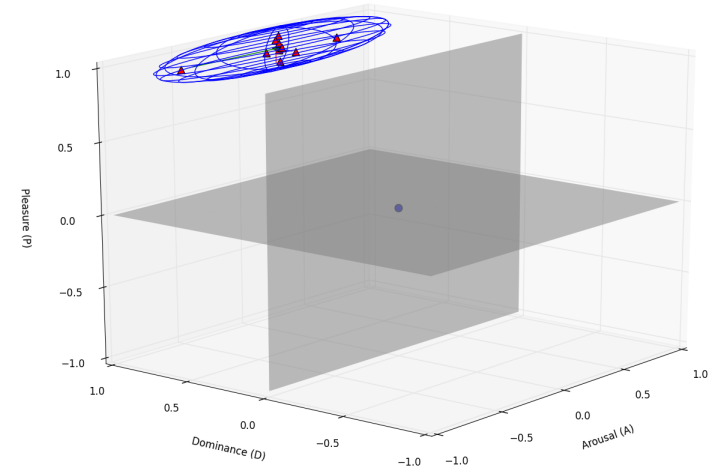

Figure 3: Representation of an ellipsoid enveloping the emotions of a group of agents.

Furthermore, considering $\vec{m}(\mathrm{Ag})$ as a part of the definition of the social emotion of a group of agents, there may be situations in which $\vec{m}(A g)$ is not enough. In Figure 4 and 5 a group of agents is shown with similar $\overrightarrow{C E}(A g)$ and $\vec{m}(A g)$, but with completely different emotional situations. In order to solve this problem the notion of standard deviation (SD) is introduced. This SD allows the calculation of the level of emotional dispersion of this group of agents around the central emotion $\overrightarrow{C E}(A g)$ for each component of the $P A D$ (Equation 8).

$$
\begin{aligned}
& \sigma_{P}(A g)=\sqrt{\frac{\sum_{i=1}^{n}\left(P_{i}-\bar{P}(A g)\right)^{2}}{n},}, \forall a g_{i} \in A g / \vec{E}\left(a g_{i}\right)=\left[P_{i}, A_{i}, D_{i}\right] \\
& \sigma_{A}(A g)=\sqrt{\frac{\sum_{i=1}^{n}\left(A_{i}-\bar{A}(A g)\right)^{2}}{n}}, \forall a g_{i} \in A g / \vec{E}\left(a g_{i}\right)=\left[P_{i}, A_{i}, D_{i}\right] \\
& \sigma_{D}(A g)=\sqrt{\frac{\sum_{i=1}^{n}\left(D_{i}-\bar{D}(A g)\right)^{2}}{n}}, \forall a g_{i} \in A g / \vec{E}\left(a g_{i}\right)=\left[P_{i}, A_{i}, D_{i}\right]
\end{aligned}
$$

\footnotetext{
${ }^{2}$ This figure is an snapshot of the emotion of a group of agents in an specific time
} 


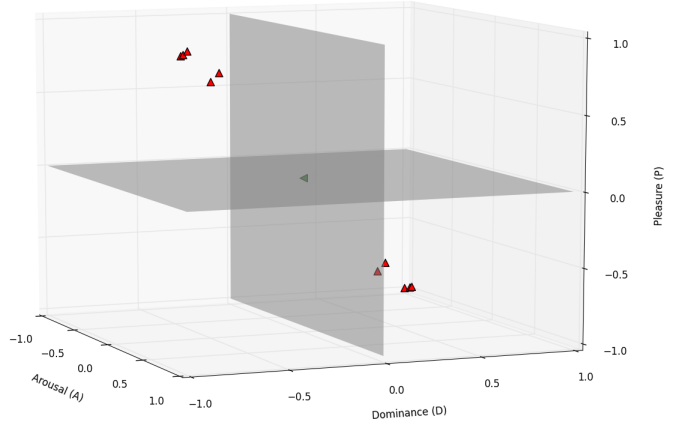

Figure 4: Representation of a group of agent's emotions with two very different subgroups

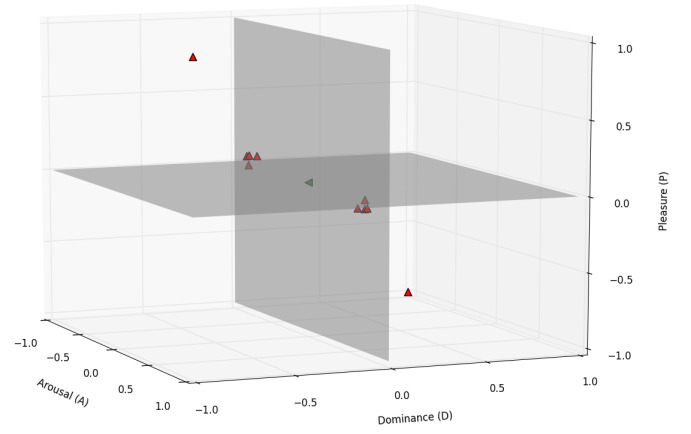

Figure 5: Representation of a group of agent's emotions with two rather different subgroups.

The result of each of the above equations can be represented as a vector (Equation 9), which allows to determine the level of emotional dispersion.

$$
\vec{\sigma}(A g)=\left[\sigma_{P}(A g), \sigma_{A}(A g), \sigma_{D}(A g)\right]
$$

The maximum value that can be represented in each one of the components of the vector $\vec{\sigma}(A g)$ is 2 . Therefore, in our case, we consider that the emotional dispersion is high when the $|\vec{\sigma}(A g)|$ is greater than 2, from this definition it can be deduced that:

1. if $|\vec{\sigma}(A g)|>>2$, the group has a high emotional dispersion, i.e. the members of the group have different emotional states. 
2. if $|\vec{\sigma}(A g)| \cong 0$, the group has a low emotional dispersion, this means that individuals have similar emotional states.

Adding the emotional dispersion in the definition of the social emotion of a group of agents, the social emotion of a group of agents $A g=a g_{1}, a g_{2}, \ldots, a g_{n}$ can be defined by the following triplet (Equation 10).

$$
S E(A g)=(\overrightarrow{C E}(A g), \vec{m}(A g), \vec{\sigma}(A g))
$$

Where $\overrightarrow{C E}(A g)$ is the central emotion, $\vec{m}(A g)$ represents the maximum distances and $\vec{\sigma}(\mathrm{Ag})$ represents the emotion dispersion of an agent group.

Based on this model it is possible to determine the emotional distance among different groups of agents or between the same group in different instants of time. This will allow to measure the emotional distance between the current social emotional group and a possible emotional target. This approach can be used as a feedback in the decision making process in order to take actions to try to move the social emotion to a particular area of the $P A D$ space or to allow that the emotional state of a group of agents can be approached or moved away from other groups of agents. From an emotional point, of view these movements or actions are domain-dependent and are out of the scope of this model. In Equation 11 the profile of the emotional distance function is defined as the distance of the social emotions of two groups of agents.

$$
\Delta_{S E}: S E\left(A g^{i}\right), S E\left(A g^{j}\right) \rightarrow[0,1]
$$

According to this profile, Equation 12 shows how we calculate this emotional variation. The equation calculates three distances corresponding to the three components of the $S E$. Given two groups of agents $A g_{i}, A g_{j}$ with social emotions $S E\left(A g^{i}\right), S E\left(A g^{j}\right)$ respectively, the emotional distance between these two groups is calculated as:

$$
\begin{array}{r}
\Delta_{S E}\left(S E\left(A g^{i}\right), S E\left(A g^{j}\right)\right)=\frac{1}{2}\left(\omega_{c} \Delta\left(\overrightarrow{C E}\left(A g^{i}\right), \overrightarrow{C E}\left(A g^{j}\right)\right)\right. \\
+\omega_{d} \Delta\left(\vec{m}\left(A g^{i}\right), \vec{m}\left(A g^{j}\right)\right) \\
\left.+\omega_{v} \Delta\left(\vec{\sigma}\left(A g^{i}\right), \vec{\sigma}\left(A g^{j}\right)\right)\right) \\
\text { where } \quad \omega_{c}+\omega_{d}+\omega_{v}=1 ; \quad \omega_{c}, \omega_{d}, \omega_{v} \in[0,1]
\end{array}
$$

and $\Delta$ calculates the vectorial distance between two vectors. As every dimension of the $P A D$ space is bounded between $[-1,1]$, each $\Delta$ will give values between $[0,2]$. Therefore, $\Delta_{S E}$ will have a range between $[0,1]$. 
Calculating the distance among social emotions allows the study of the behavior of emotional-based agents, either minimizing or maximizing the $\Delta_{S E}\left(S E\left(A g^{i}\right), S E\left(A g^{j}\right)\right)$ function. This way, it can be achieved that an agent group approaches or move away of an specific emotional state. To do this it is necessary to modify through stimuli the individual emotions from each agent and therefore changing the social emotion. Nevertheless, how to maximize or minimize the emotional distance is domain-dependent and it is out of the scope of this paper.

\section{Case Study}

A practical application which uses the previously proposed model is presented in this section. This application example is based on how music can influence in a positive or negative way over emotional states [8], [18], [19].

The application example is developed in a bar, where there is a DJ agent in charge of playing music and a specific number of individuals listening the music. The main goal of the DJ is to play music making that all individuals within the bar are mostly as happy as possible. Each of the individuals will be represented by an agent, which has an emotional response according to its musical taste. That is, depending on the musical genre of the song, agents will respond varying their emotional state. Moreover, varying emotions of each agent will modify the social emotion of the group.

In such a way, the proposed application seeks to identify the different emotional states using them as a tool of communication between humans and agents. To perform this detection we need to use pattern recognition algorithms and image and audio processing techniques in order to detect and classify the different emotional states of humans. The application has been developed as a virtual multi-agent system using the JaCalIVE framework [20] where there will be different entities. Each one of these entities may represent not only real or simulated human beings, and the DJ agent, but also the furniture or the speakers with their location. The application is composed of two types of entities, a DJ agent which is the responsible of playing the music and different agents that represent humans who are inside the bar (see Figure 6). The main tasks of the different type of agents are:

- DJ agent: the main goal of this agent is to achieve a emotional state of happiness for all of the people which are in the bar. When the DJ agent plays a song, it must analyze the emotional state of people. According 
to this analysis it will select the most appropriated songs in order to improve, if possible, the current emotional state of the audience.

- Human-immersed agent: it is in charge of detecting and calculating the emotional state of an individual which is in the bar, sending this information to the DJ agent. In order to accomplish its tasks, this agent must have access to a variety of input/output information devices as cameras, microphones, ...

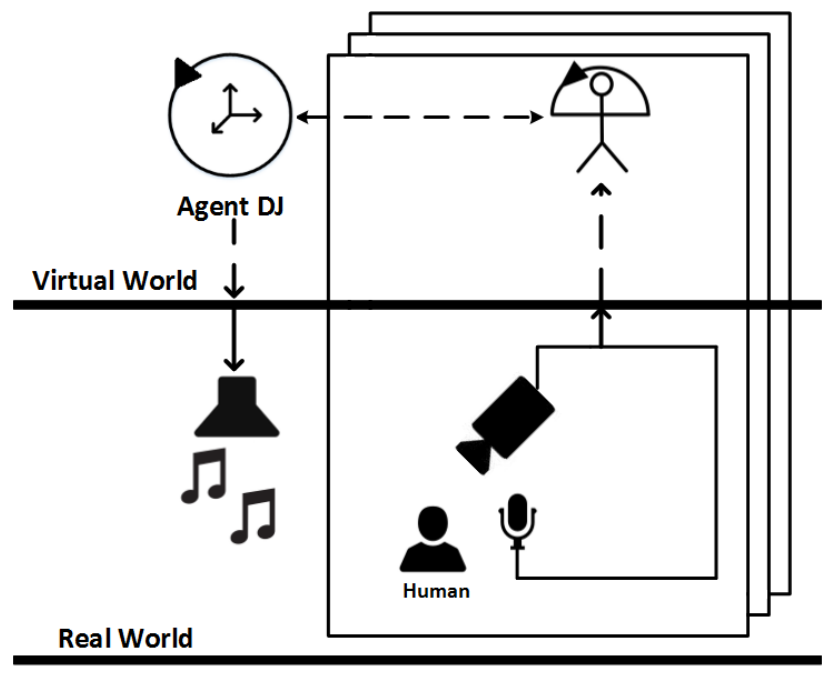

Figure 6: Simple scheme of the application

\subsection{Analysis of possible scenarios}

Two different scenarios have been designed in order to illustrate how the social emotion can facilitate the decision making of the DJ. In each scenario the DJ agent plays a song. Once the song has ended, the DJ evaluates the social emotion of the group of listeners that are within the bar. In this way, the DJ agent can evaluate the effect that the song has had the song over the audience. This will help the DJ to decide whether to continue with the same musical genre or not in order to improve the emotional state of the group. 


\subsubsection{Scenario 1: Group of agents with a low emotional dispersion}

The first case analyzed is one in which the emotional states of the agents are close. This emotional difference may be due mainly because the agents have little differences in their musical tastes. The social emotion in this scenario has a $\overrightarrow{E C}(A g)$ very close to all the values of the agents and the $\vec{m}(A g)$ and $\vec{\sigma}(A g)$ values will be very small and in many cases close to zero. This provokes that the DJ will try to play songs of similar generes trying to maintain this situation, which is not the ideal situation but it can be considered as a very good situation. A graphical representation of this example can be seen in Figure 7 while Table 1 shows the different emotional states of each of the agents in this group.

Table 1: Individual emotion of each agent and its magnitude in the PAD space

\begin{tabular}{ccccc}
\hline \hline Agents & P & A & D & Emotional State \\
\hline ag_0 & 0.90 & 0.0 & 0.90 & Happy \\
ag_1 & 0.70 & 0.0 & 0.91 & Happy \\
ag_2 & 0.80 & 0.0 & 0.95 & Happy \\
ag_3 & 0.85 & 0.0 & 0.99 & Happy \\
ag_4 & 0.91 & 0.0 & 0.89 & Happy \\
ag_5 & 0.93 & 0.0 & 0.86 & Happy \\
ag_6 & 0.89 & 0.0 & 0.83 & Happy \\
ag_7 & 0.79 & 0.0 & 0.81 & Happy \\
ag_8 & 0.92 & 0.0 & 0.89 & Happy \\
ag_9 & 0.81 & 0.0 & 1.0 & Happy \\
\hline
\end{tabular}

As it can be see in the Figure 7 all the represented emotions in this group are around the emotion Happy, achieving a social emotion with these values of $S E(A g)=([0.85,0.0,0.9],[0.85,0.0,0.9],[0.07,0.0,0.06])$.

\subsubsection{Scenario 2: Group of agents with high emotional dispersion}

In this second case it is represented the existence of a group of agents emotionally dispersed in the bar. These agents have completely different emotions distributed along the $P A D$ space. The emotional values of each of the agents can be seen in Table 2. This high dispersion is reflected in the calculated values of the social emotion:

$S E(A g)=([0.14,0.42,0.1],[1.14,1.32,1.0],[0.87,0.67,0.79])$

In this case, the social emotion is very different and more complicated to manage than in the previous case. The central emotion is very far from the emotional states of each agent and the maximum distances and dispersion values are high too. So, from the perspective of the DJ this scenario is very chaotic and unwished because it is difficult to choose which kind of 


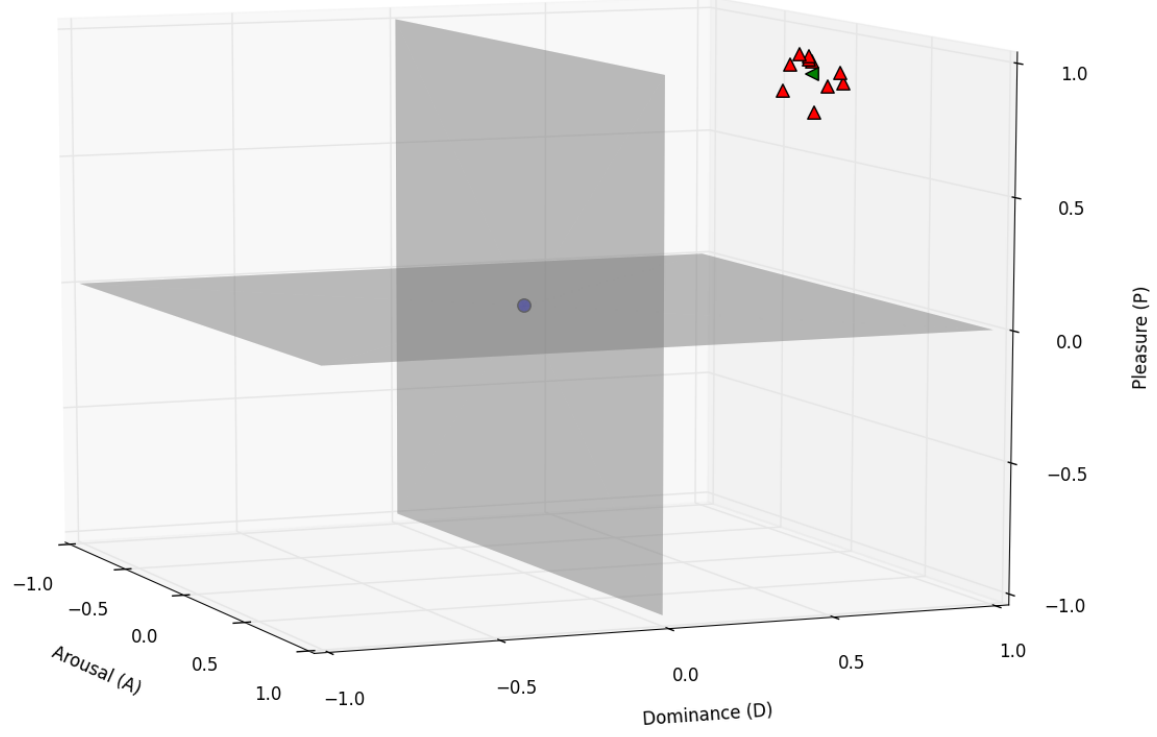

Figure 7: Scenario 1: Representation of the emotional states of a group of agents with a low emotional dispersion.

music is the most appropriated. In this case the DJ should try to move the central emotion to a state close to "happy" testing different musical styles and analyzing carefully the effect of each song in the social emotion of the group.

Table 2: Individual emotion of each agent and its magnitude in the PAD space

\begin{tabular}{ccccc}
\hline \hline Agents & P & A & D & Emotional State \\
\hline ag_0 & -0.9 & -0.9 & -0.9 & Remorse \\
ag_1 & -0.7 & 0.6 & 0.0 & Anguish \\
ag_2 & 0.9 & 0.9 & 0.0 & Joy \\
ag_3 & 0.9 & -0.5 & 0.9 & Satisfaction \\
ag_4 & -0.7 & 0.8 & -0.9 & Hurt \\
ag_5 & 0.9 & 0.9 & 0.9 & Admiration \\
ag_6 & 0.9 & 0.0 & 0.9 & Happy \\
ag_7 & -1.0 & 1.0 & 0.9 & Anger \\
ag_8 & 1.0 & 1.0 & -0.9 & Love \\
\hline
\end{tabular}

\subsection{Improving by learning of the DJ agent}

The above commented application is formed by two different types of agents: the DJ Agent and the Human-Immersed Agent. Regarding the DJ 


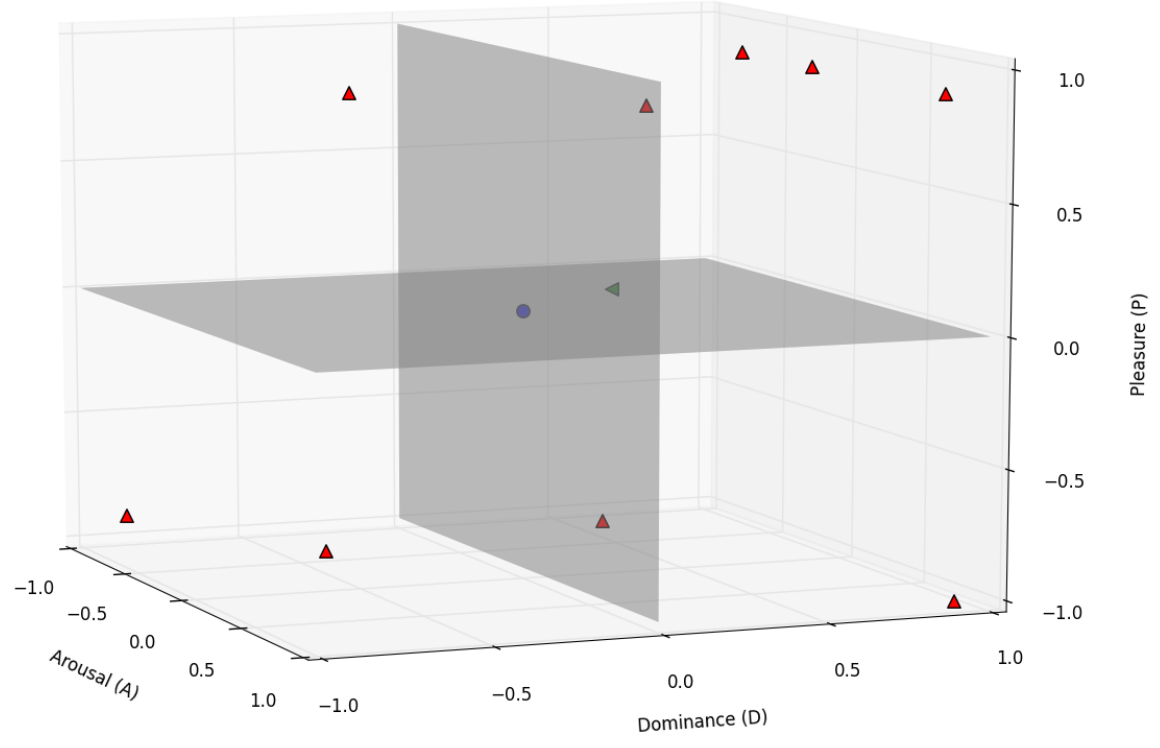

Figure 8: Scenario 2: Representation of the emotional states of a group of agents with a high emotional dispersion.

Agent, this agent uses the information sent by the Human-Immersed Agent to analyze the group's emotional state, using it to decide which is the following song to play. Obviously, the goal of the DJ Agent is that all the humans feel as happy as possible. To achieve this, each of the agents representing humans should communicate previously their emotional state. For doing this, it is necessary to provide each Human-Immersed Agent with a serie of sensors which generate enough information to be able to know the emotional state of each human. After this, the DJ agent calculates the social emotion and decides the next song. As this decision will affect in the emotional state of people, it is necessary to include some kind of mechanism to facilitate this decision.

The mechanics selected to give the $D J$ agent the capability of learning and predicting the new social emotion was a neural network $(A N N)$. Since it not only has the capability of classifying but it also has the ability to predict[21]. This ability is used to predict the social emotion caused by an specific song played in the bar. The main process is defined as follows: 1) 


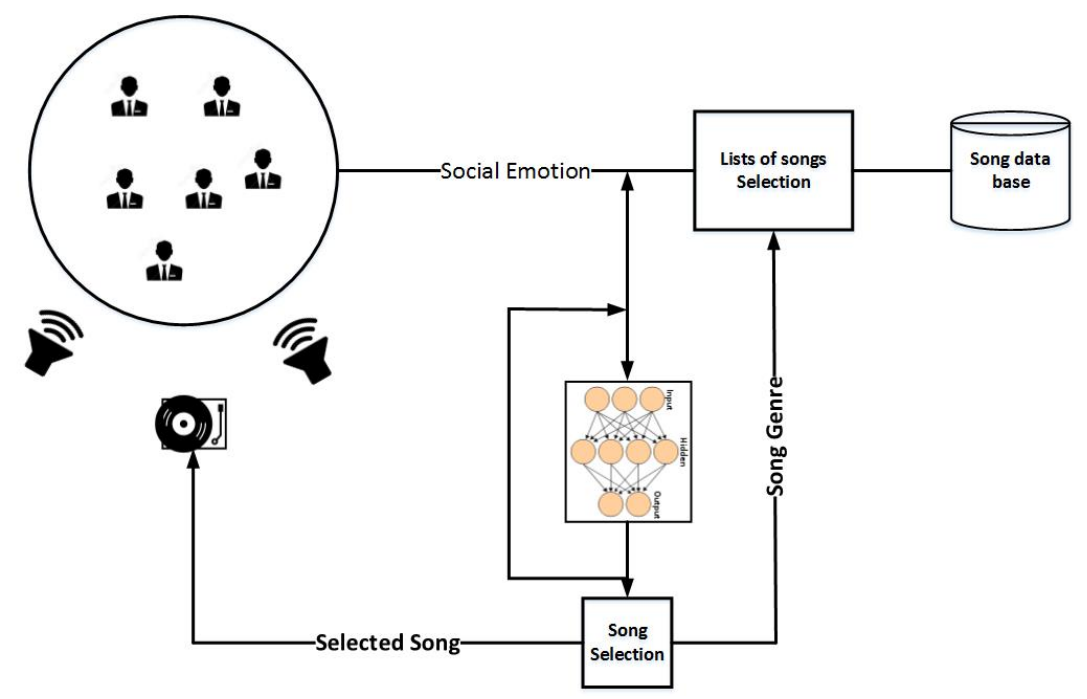

Figure 9: Predictive System Scenario

the social emotion of the group is calculated using the information provided by the Human-Immersed Agents; 2) An ANN is used to predict the future social emotion of people taking into account the previously calculated current social emotion and a set of songs, which are similar to the previously played song (this is done searching by genre in the database of songs); 3) the song which minimizes the distance between the target emotion (happiness) and the predicted social emotion is selected; and 4) the selected song is played in the bar and the process begins again. A graphical view of the application process is shown in Figure 9.

The ANN's architecture used is a Background Propagation as it is the most used and gives the best results for making predictions [22]. Figure 10 shows the architecture of the proposed $A N N$, which is composed of: 10 input neurons, 5 neurons in the middle layer and 9 output neurons. The ten inputs of the neural network are the current social emotion and a musical genre. The output layer is the social emotion predicted by the $A N N$ for the group of agents.

Different sessions were created in order to train our $A N N$ with different personalities and musical preferences of the audience. The results of these simulations were stored in order to create a dataset. For each iteration, the previous social emotion, the played song, the musical genre and the 
social emotion calculated after the song were stored. Once the simulations were completed, the generated data was used for the training process of the proposed $A N N$.

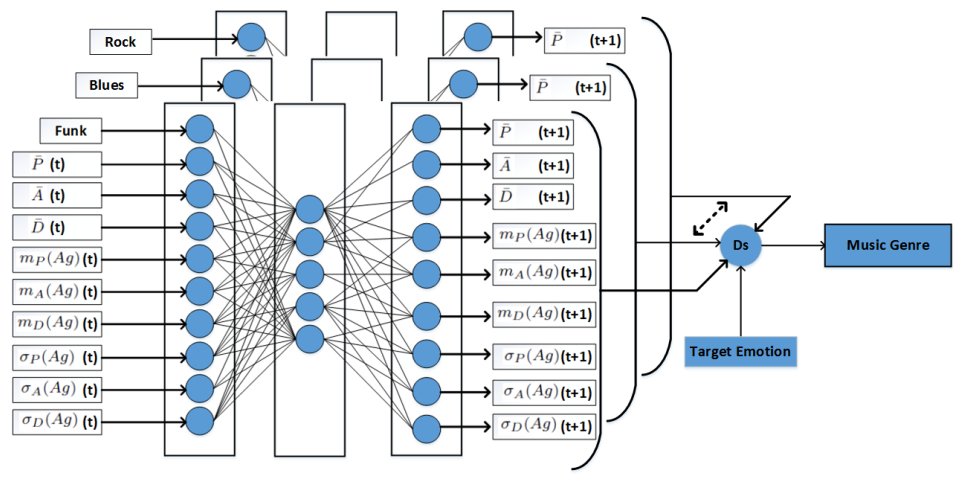

Figure 10: Neural Network Architecture

The $A N N$ was trained using a supervised trained methodology, since the objective of the network is to predict the future value of the social emotion after a specific song is played. Concretely, the training process employed a dataset composed by 3000 entries. This information was extracted from 5 simulated sessions. In each one of the sessions, the parameters of each agent were modified changing their personality values (defined according to the OCEAN Model [23]) and their musical preferences. From the obtained dataset, the $25 \%$ was used to test and the $75 \%$ was used to train. Figure 11 shows the generated main square error in each iteration of the training process.

Moreover, the results generated by the proposed ANN have been compared with two simple methods. The first one considers that the predicted social emotion is the same than the previous one. The second one predicts as the new social emotion the average of the last ten social emotions calculated. Table 3 summarizes the obtained results for each method, showing the MSE calculated individually for each PAD value. These $M S E$ values calculated in the three experiment allow us to compare which of the three experiment gives the best results.

As can be seen, the ANN achieves better results with lower MSE values for each component of the $P A D$ vector.

In order to analyze the effect of the ANN in the proposed application, 


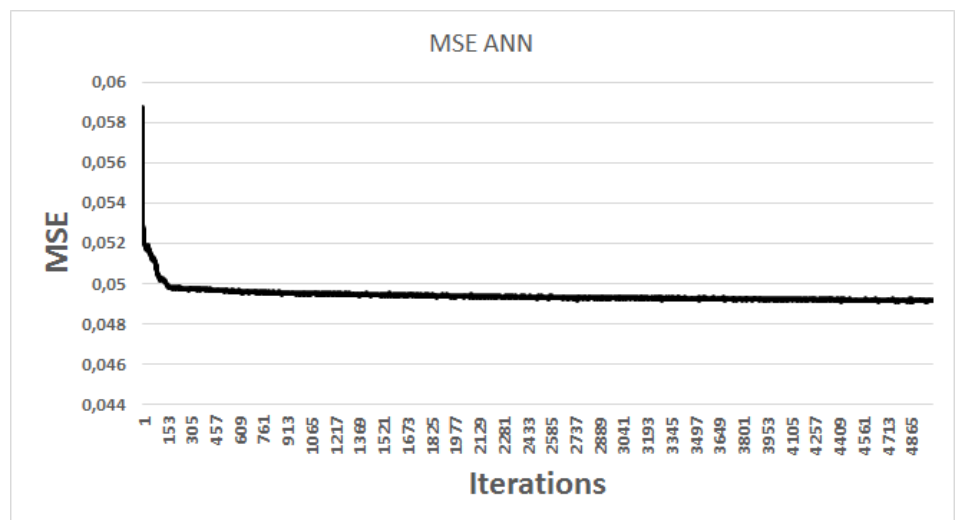

Figure 11: Evolution of the Mean Square Error (MSE) of the Neural Network

\begin{tabular}{|l|l|l|l|}
\hline Experiment & MSE P & MSE A & MSE D \\
\hline ANN & 0.006337 & 0.0082748 & 0.01309546 \\
\hline Simple 1 & 0.0503843 & 0.0086129 & 0.5056521 \\
\hline Simple 2 & 0.0463723 & 0.0084158 & 0.5057954 \\
\hline
\end{tabular}

Table 3: Mean Square Error obtained for each PAD component

different experiments have been developed. Specifically, the aim of these tests is to validate the use of the ANN as a way to predict the social emotion of a group of agents and, thus, improve the decision making process. To do this, the implemented prototype has been tested changing the music played in the bar (around 50 songs are played in an iterative way) and each test has been repeated 100 times. All the tests are composed by agents representing people which is in the bar. These agents have assigned random initial emotions at the beginning of the execution of each test.

Figures 12 and 13 represent the evolution of the dispersion around the group of agents' social emotion in two different configurations of the system. In the first one, the songs played are randomly selected among the set of songs of the same genre. In the second one, the application selects the songs to be played using the previously exposed process including the proposed ANN. The dispersions showed in the Figures are calculated separately for each component of the PAD vector. As it can be observed, as time passes the dispersion is lower in the case of the experiment which includes the ANN as a prediction tool. This fact is especially remarkable in the case of the 


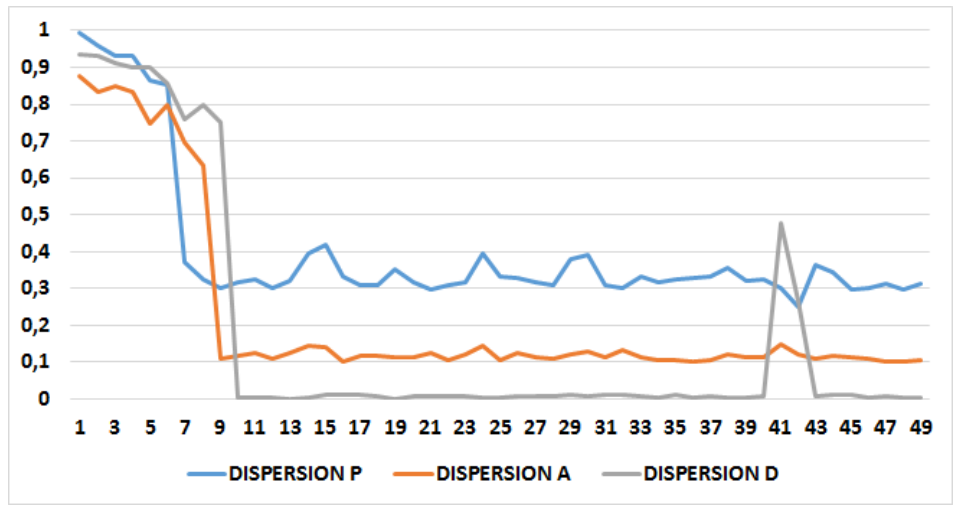

Figure 12: Evaluation of the maximum dispersion of the social emotion for each PAD component (without the ANN)

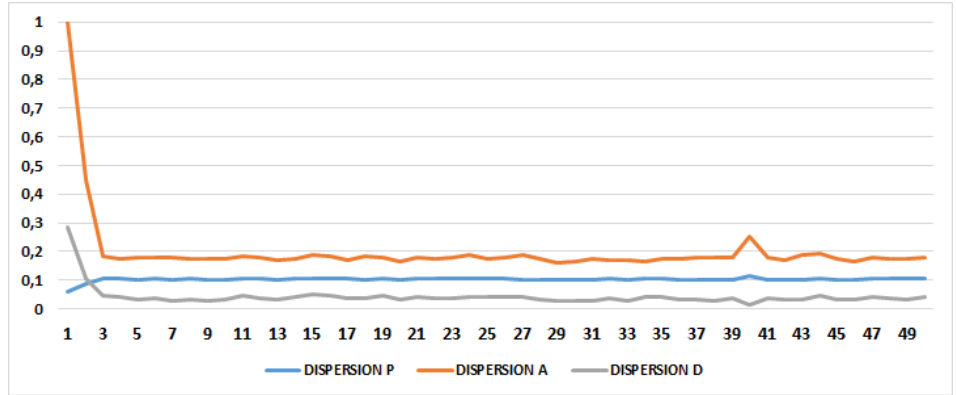

Figure 13: Evaluation of the maximum dispersion of the social emotion for each PAD component (with the ANN)

Pleasure $(\mathrm{P})$ component, because it is the most related component regarding happiness. The achieved dispersion in the Pleasure component is extremely lower when we employ the ANN.

Finally, Figure 14 shows the results obtained regarding the distance between the social emotion of the group and the target emotion (Happy). As we can see, the system quickly reduces the distance of the social emotion with regard to the target emotion. After few iterations, the distance remains constant around 0.5 , which can be considered a very good and close approximation. 


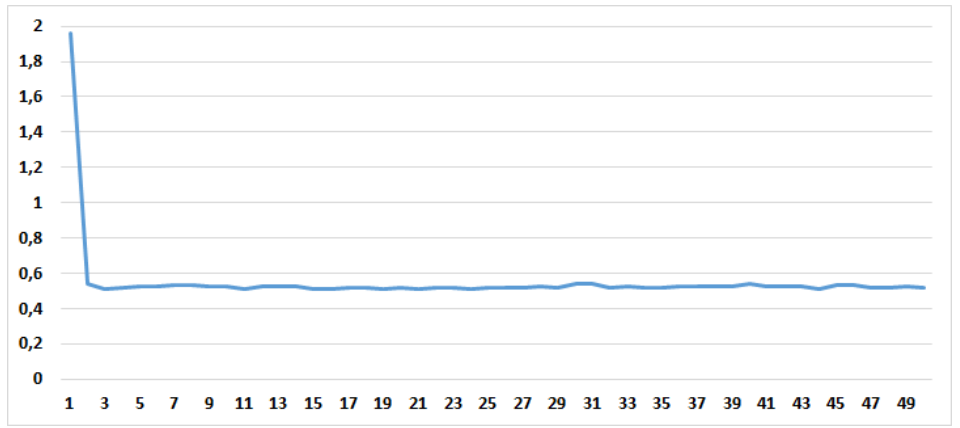

Figure 14: Evaluation of the distance between the social emotion against happiness

\section{Conclusions and future work}

A new model for representing social emotions has been presented in this paper. The goal of this model is to give a first approach for the detection and simulation of social emotions in a group of intelligent entities. This social emotion model builds on the PAD emotional model, which allows the representation of individual emotions in intelligent entities. The proposed model of social emotion uses the individual emotions of each entity of a group, allowing us to represent the emotion of that group as a triplet consisting of three vectors $(\overrightarrow{E C}(A g), \vec{m}(A g)$ and $\vec{\sigma}(A g))$. This definition allows us to represent the emotional state of a group of entities that are placed in a specific environment. Moreover, the model adds the mechanisms to compare the social emotional state of two groups of agents or the social emotion of a group in different time instants. The social emotion of a group of agents not only allows a global view of the emotional situation of the group, moreover it can be used as a feedback in order to change the emotional state of the group or only of a part of the agents.

The paper also presents an application able to extract (in a non-invasive way) and to analyze the social emotion of a group of persons and it takes decisions according to that emotional state. The application includes a learning module which will allow to predict future emotional states of the people. According to the obtained results, this module improves the decision making of system comparing the social emotional values that are obtained during the execution of the system.

The proposed model can be also applied to many different domains. Some of them, that we would like to underline, include big systems simulations in 
ambient intelligence in order to predict human behavior, even in industrial environments, where it can monitor and simulate the human conditions inside a factory. Moreover, in the robotics domain, this model can be used to model and simulate the emotions of the individuals that interact with the robots allowing an improvement of the accuracy of the robot's decision-making.

As future work we want to introduce the human within the model, adding their emotional state through the analysis of body gestures or through the face. To do this, specialized hardware must be used in order to obtain this information, helping to create environments in which humans interact in a transparent way with intelligent entities employing their emotional states.

\section{Acknowledgements}

This work is partially supported by the MINECO/FEDER TIN201565515-C4-1-R and the FPI grant AP2013-01276 awarded to Jaime-Andres Rincon.

\section{References}

[1] S. Tivatansakul, M. Ohkura, Healthcare system design focusing on emotional aspects using augmented reality, in: 2013 \{IEEE\} Symposium on Computational Intelligence in Healthcare and e-health (\{CICARE\}), 2013, pp. 88-93. doi:10.1109/CICARE.2013.6583074.

[2] D. O. Bos, \{EEG\}-based emotion recognition, The Influence of Visual and Auditory Stimuli (2006) 1-17.

[3] T. D. Pham, D. Tran, Emotion Recognition Using the Emotiv $\{$ EPOC Device, in: T. Huang, Z. Zeng, C. Li, C. S. Leung (Eds.), Neural Information Processing, no. 7667 in Lecture Notes in Computer Science, Springer Berlin Heidelberg, 2012, pp. 394-399.

[4] P. A. Nogueira, R. Rodrigues, E. Oliveira, L. E. Nacke, A hybrid approach at emotional state detection: Merging theoretical models of emotion with data-driven statistical classifiers, in: 2013 IEEE WIC ACM International WI and IAT, Vol. 2, 2013, pp. 253-260.

[5] I. T. Meftah, N. L. Thanh, C. Ben Amar, Towards an algebraic modeling of emotional states, in: 2010 Fifth International Conference on 
Internet and Web Applications and Services (ICIW), 2010, pp. 513-518. doi:10.1109/ICIW.2010.82.

[6] A. Ortony, G. Clore, A. Collins, Cognitive Structure of Emotions, Cambridge University Press, 1988.

[7] a. Mehrabian, Analysis of affiliation-related traits in terms of the PAD Temperament Model., The Journal of psychology 131 (1) (1997) 101117. doi:10.1080/00223989709603508.

[8] B. Whitman, P. Smaragdis, Combining Musical and Cultural Features for Intelligent Style Detection., in: Ismir, Paris, France, 2002, pp. 5-10.

[9] C. Rea, P. MacDonald, G. Carnes, Listening to classical, pop, and metal music: An investigation of mood, Emporia State Research Studies 46 (1) (2010) 1-3.

[10] W. F. Thompson, L. Quinto, Music and emotion: Psychological considerations, The Aesthetic Mind: Philosophy and Psychology (2011) $357-375$.

[11] Council on Communications and Media, Impact of Music, Music Lyrics, and Music Videos on Children and Youth, PEDIATRICS 124 (5) (2009) 1488-1494. doi:10.1542/peds.2009-2145.

[12] C. Becker-Asano, I. Wachsmuth, Affective computing with primary and secondary emotions in a virtual human, Autonomous Agents and MultiAgent Systems 20 (1) (2010) 32-49.

[13] D. Jain, Z. Kobti, Simulating the Effect of Emotional Stress on Task Performance Using OCC, in: C. Butz, P. Lingras (Eds.), Advances in Artificial Intelligence, no. 6657 in Lecture Notes in Computer Science, Springer, 2011, pp. 204-209. doi:10.1007/978-3-642-21043-3_24.

[14] A. Ortony, B. N. Colby, A. Ortony, G. L. Clore, A. Collins, The Cognitive Structure of Emotions, no. 6, Cambridge University Press, 1990. doi: $10.2307 / 2074241$.

[15] F. Ali, M. Amin, The influence of physical environment on emotions, customer satisfaction and behavioural intentions in Chinese resort hotel industry, in: J. for Global Business Advancement, Vol. 7, 2013, pp. 249 $-266$. 
[16] A. Mehrabian, Comparison of the PAD and PANAS as models for describing emotions and for differentiating anxiety from depression, Journal of psychopathology and behavioral assessment 19 (4) (1997) 331-357.

[17] A. Mehrabian, Basic Dimensions for a General Psychological Theory: Implications for Personality, Social, Environmental, and Developmental Studies, year $=1980$, Oelgeschlager, Gunn \& Hain.

[18] M. D. van der Zwaag, J. H. D. M. Westerink, E. L. van den Broek, Emotional and psychophysiological responses to tempo, mode, and percussiveness, Musicae Scientiae 15 (2) (2011) 250-269. doi:10.1177/1029864911403364.

[19] K. R. Scherer, M. R. Zentner, Emotional Effects of Music : Production Rules, Music and emotion: Theory and research (2001) 361-392.

[20] J. A. Rincon, C. Carrascosa, E. Garcia, Developing intelligent virtual environments using mam5 meta-model, in: Advances in Practical Applications of Heterogeneous Multi-Agent Systems. The PAAMS Collection, Springer, 2014, pp. 379-382.

[21] B. OANCEA, S. C. CIUCU, S. C. Ciucu, Time series forecasting using neural networks, Proceedings of the CKS 2013 International Conference (2003) 1402-1408arXiv:1401.1333.

[22] D. Nayak, A. Mahapatra, P. Mishra, A Survey on Rainfall Prediction using Artificial Neural Network, International Journal of Computer ... 72 (16) (2013) 32-40.

[23] A. Egges, S. Kshirsagar, N. Magnenat-Thalmann, Generic personality and emotion simulation for conversational agents, Comp. Anim. Virtual Worlds 15 (1) (2004) 1-13. doi:10.1002/cav.3. 